\title{
Personal branding y personal marketing: procesos complementarios centrados en la marca personal
}

Personal branding and personal marketing: complementary processes focused on the personal brand

\begin{tabular}{|l|}
\hline Fabiola Patricia \\
Melchor Rueda \\
Lean Business Group, \\
México
\end{tabular}

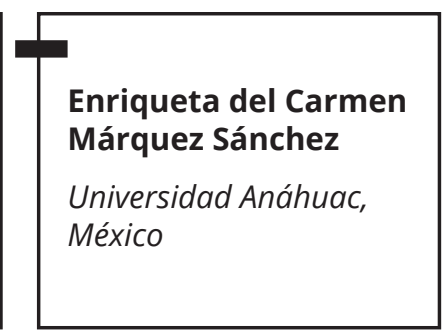

Vladimir Deléyade
Estrada Portales
Universidad
Dominicana O\&M,
República Dominicana

Recibido: 23 de julio de 2021.

Aprobado: 23 de noviembre de 2021. 


\section{Resumen}

El presente artículo aborda la marca personal como concepto, atributo y resultado humano, como categoría a gestionar para lograr un posicionamiento personal y profesional favorable en un entorno cada vez más cambiante, exigente y competitivo, así como los dos procesos esenciales para ello: el personal branding y el personal marketing.

El objetivo es analizar los tres conceptos y sus puntos de contacto, a partir de la forma en que se definen y perciben, mediante una investigación bibliográfica y un estudio exploratorio, en los que se profundiza en la percepción y el conocimiento que se tiene de la marca personal, a través de una encuesta a 112 mexicanos, con la que se comprueba cómo las personas tienen una idea de lo que es la marca personal, sin poder describirla adecuadamente. Se proponen posiciones sobre la importancia de este concepto y su aplicación correcta, para las organizaciones y la sociedad en la época actual.

Con base en los contenidos revisados, se define y analiza la marca personal, el personal branding y el marketing personal, se identifican y valoran las posturas comunes, y se procura la integración analítica de los tres conceptos.

Palabras clave: marca personal, personal branding, personal marketing, organizaciones, posicionamiento, liderazgo, expertos.

Clasificación JEL: M39, M59.

\section{Abstract}

The present article addresses the Personal Brand as a concept, attribute and human result, as well as a category to be managed in order to achieve a favorable personal and professional positioning in an increasingly changing, demanding and competitive environment. It also addresses the two essential processes to achieve it: personal branding and personal marketing.

The objective is to analyze the three concepts and their intersecting points, based on the way in which they are defined and perceived through a bibliographic research and an exploratory research, in which the perception and knowledge of the personal brand is deepened, through a survey applied to 112 Mexicans, which allowed us to verify that people do have an idea of what personal branding is, without being able to describe it adequately. Positions are proposed on the importance of this concept and its correct application, for both organizations and society in the current era.

Based on the reviewed content, the personal brand, personal branding and personal marketing are defined and analyzed, common postures are identified and valued, seeking the analytical integration of the three concepts.

Keywords: personal brand, personal branding, personal marketing, organizations, positioning, leadership, experts.

JEL Classification: M39, M59. 


\section{Introducción}

La época emergente exige nuevos modos de actuación y relacionamiento, y nuevos estándares de desempeño humano, en cualquier entorno.

Ese conjunto de interacciones, interinfluencias e impactos entre personas es objeto de investigación y otras intervenciones desde hace 25 años, bajo el concepto de marca personal. Esta es una categoría cuya esencia y práctica devienen fuentes de desarrollo personal, organizacional y social, partiendo del vínculo entre las personas, sus desempeños y los resultados que aportan a las organizaciones donde producen bienes o prestan servicios de valor para la sociedad, sea con alcance individual o colectivo.

Las personas que construyen su marca personal, solas o a través de un experto en personal branding y personal marketing, son capaces de convertirse en marcas personales poderosas, modélicas, relevantes, transformadoras. Sobre este concepto, su pertinencia y su impacto, trata el presente artículo.

El concepto de marca personal toma cuerpo y relevancia a partir de un artículo icónico llamado «La marca llamada Usted», donde se sugiere a las personas aprender las lecciones de las marcas de las grandes empresas y aplicarlas en su vida para prosperar en el mundo del trabajo (Peters,1997). Posteriormente, surgen libros donde se dan numerosas ideas y recomendaciones para que las personas puedan hacer una marca de su persona (Peters,1999). Ciertos autores indican que esto comenzó a utilizarse antes, y que siempre ha existido, solo su definición conceptual es reciente. Por ejemplo, Pérez Ortega (2008) menciona que existe desde que el hombre es hombre. Destaca que, si uno tiene algo que ofrecer y otra persona tiene una necesidad, se puede realizar un intercambio, pero antes debe conocerse a la persona y confiar en ella.

Este artículo propone un acercamiento conceptual y analítico a los procesos de gestión y mercadotecnia de la marca personal, también conocidos como personal branding y personal marketing, desde las miradas de las investigaciones de diversos autores y los hallazgos de un sondeo exploratorio, con el objetivo de conectar los tres conceptos en una lógica sistémica, y analizar algunas ideas relacionadas con estos que se han extendido en la sociedad y el mercado. 


\section{Marco teórico}

Hoy en día, se puede plantear que sobre la marca personal existe un cuerpo doctrinal propio a nivel teórico, metodológico, ético e instrumental, que abarca las diversas formas de implementar el concepto y gestionar su práctica; y cuya génesis puede encontrarse, al menos parcialmente, en las concepciones sobre la marca como categoría de mercadotecnia.

De acuerdo con Fernández et al. (2018), los planteamientos relativos a la marca como categoría general, resultan plenamente aplicables a la marca personal, como ámbito específico de aplicación y gestión de esa categoría.

A continuación, se analizan diferentes aspectos sobre la marca personal, las formas de construirla y desarrollarla, que dan cuerpo y sustento a la propuesta del presente artículo.

\section{La marca personal y su proceso de gestión}

Es importante destacar que cuando nos referimos a personas, la marca se considera la huella de carácter moral que se deja en los demás al momento de relacionarse e interactuar (Real Academia Española, 2014). Por esta y otras razones, resulta válido y plausible asumir y practicar la combinación interconceptual entre marca y personas, tanto en un sentido estratégico como operativo.

Esta lógica es compartida por diversos autores, que coinciden con el carácter relacional e interpersonal de la marca personal, desde varias de sus dimensiones, entre ellas, autenticidad, diferencia, influencia e impacto. Cada ser humano deja una huella diferente, debido a que cada individuo posee cualidades distintas y únicas en relación con otras personas (Buitrago y León, 2018).

La construcción y desarrollo de la marca personal no significa diseñar y aplicar una estrategia egocéntrica sino, más bien, la búsqueda de las características distintas de cada persona, según parámetros de autenticidad y coherencia, que se ponen al servicio de todos (Molares-Cardoso et al., 2020).

Los elementos que forman la marca personal del individuo son la apariencia física, el comportamiento, el mensaje, el networking o el tipo de relaciones que establece con otras personas y su presencia en internet. Aunque el surgimiento del concepto 
propiamente dicho data de 1997, existen evidencias anteriores sobre el análisis del tema.

Peters (1997) propuso a los humanos como marcas para una audiencia más amplia. Para estas marcas humanas, considera que cada individuo posee una, aunque la gente normalmente no la conoce. Así, esta se convierte en esa huella para otras personas, quienes la asumen y posicionan como esa marca de la persona, diferente a cualquier otra (Ries y Ries, 2001; Estrada, 2020a). Con una gestión inteligente de su marca personal, es posible generar condiciones que puedan propiciar un posicionamiento favorable, sea por sus comportamientos como persona y ser social, sea por sus desempeños como profesional, bien por los logros que obtiene integrando ambas dimensiones o por lo que ocurre en las tres esferas y en su integración.

Como puede apreciarse, la aplicación del concepto marca a las personas y su actividad permite identificar claramente dos ámbitos o dimensiones: la personal y la profesional, en ambos casos con incidencia en lo social. No obstante, como se verá más adelante, estas no deberían separarse para su análisis y gestión, porque constituyen y proyectan una entidad única, integrada y gestionable desde lo sistémico (Sagredo, 2012; Estrada, 2020b).

Es posible que la persona pueda ser conocida, reconocida, memorable y elegida (Collell, 2019), no solo por lo que demuestra ser como profesional, sino también por la calidad humana que se manifiesta en sus desempeños profesionales.

En este orden de ideas, Park et al. (2020) enfatizan la importancia de la marca personal, afirmando que cualquier persona puede desarrollar una que resulte fuerte, posicionándose de maneras únicas que los separan de los demás en el contexto de la competencia por puestos de trabajo.

Rampersad (2009) considera que la marca personal es el conjunto de percepciones, imágenes y expectativas creadas en los pensamientos de otros, mientras que para Acosta (2006), la marca personal va estrechamente ligada al apego emocional de las personas: cuando generamos emociones positivas en otros seres humanos, como empatía, confianza, autenticidad, credibilidad, valores compartidos u otros, es mucho más fácil que un cliente vuelva a trabajar contigo.

Para Balula y Vasconcelos (2020), la marca personal puede definirse como las creencias, valores, atributos y características de un ser humano, que generan una diferenciación en las mentes de otras personas a las que se quiere impactar, que es resultado de lo que hacen los individuos y cómo lo hacen. 
Esta lógica integra la dimensión interna y la proyección externa de la marca personal: impactar el entorno desde el ser y el accionar propios, esto último integrado por el comportamiento personal y el desempeño profesional, para obtener el tipo y nivel de logro que se desea y espera, tanto en una dimensión como en la otra, y en la integración de ambas. Con ello coincide Carrillo (2021) cuando propone que para desarrollar la marca personal es importante darle notoriedad, a través de diferentes acciones estratégicas (Peters, 1997).

De acuerdo con Peters (1997), para el desarrollo del personal branding podemos considerar tres etapas: la primera, enfocada en la evaluación personal y profesional del individuo; la segunda, centrada en la construcción del posicionamiento de la persona y, la tercera, dedicada a comunicar los atributos seleccionados. Adicionalmente, Pérez Ortega (2008) propuso un modelo que también consiste de tres fases:

1. Análisis de las principales características y cualidades de la persona (atributos).

2. Crear estrategias diferenciadoras de otros individuos (diferenciación).

3. Dar a conocer los principales atributos seleccionados para la creación de la marca personal (notoriedad).

Todas las personas, al expresar su personalidad, mantienen y establecen relaciones con otros individuos de manera espontánea, mientras que, al usar las herramientas de creación de marca en la persona, se establecen relaciones con los clientes o personas objetivo a los que se busque impactar (Regalado-Ortegón, 2018).

\section{Personal branding}

El proceso intencional y estratégico de gestión que se efectúa para construir una marca personal es conocido como personal branding. Es, esencialmente, la manera estratégica de construir la marca personal, para dejar huella en otras personas. Su significado es:

Identificar y comunicar las características que nos hacen sobresalir, ser relevantes, diferentes y visibles en un entorno homogéneo, competitivo y cambiante, o el conjunto de acciones planificadas que vamos a realizar para generar experiencias que produzcan una impresión positiva en tu audiencia. Un método, sistema o proceso planificado para conseguir que la gente te conozca, sepa lo que haces y obtengas el reconocimiento que mereces (Pérez Ortega, 2014, 74). 
Personal branding se define como:

Un proceso que tiene sus orígenes en terrenos propiamente organizacionales, como el marketing, la comunicación corporativa y el propio branding. Por lo tanto, implica la aplicación de metodologías, herramientas, técnicas y experiencias de dichas disciplinas en la gestión de los profesionales. El principal objeto de dicha gestión es concretamente la marca personal, que de manera similar a las marcas tradicionales implica el posicionamiento, la diferenciación y los valores que una persona proyecta y construye en su entorno a través de sus interacciones, actos, uso de los medios sociales (Del Toro, 2015).

Nótese el énfasis en el componente instrumental del proceso: se trata de hacer cosas, basadas en conceptos, aplicando métodos y técnicas en ciertos entornos, para obtener determinados resultados previamente proyectados para ser conocido y recordado de la mejor manera. Y esto es válido para cualquier generación y edad.

Por su parte, Recolons (2020) lo propone como la forma en que una persona construye su marca para crear valor y conseguir objetivos medibles. La construcción de la marca de una persona es una línea continua; es un compromiso de por vida, el de invertir en uno mismo de acuerdo con el valor que se aporta a la comunidad, y también es un modelo que inicia con establecer maneras para que las personas se conozcan a sí mismas, detecten sus fortalezas y, con base en las mismas, desarrollen las estrategias para la construcción de su marca personal; y finalmente se establece todo el plan de comunicación y selección de medios para dar notoriedad a su marca personal, proceso que arranca con el diagnóstico de marca personal o autoconocimiento (Recolons, 2021a).

En este orden de ideas, Pérez y Gringarten (2020) proponen que todas las personas poseen una marca personal, lo sepan o no. Comprender lo que puede hacer una marca ayudará a las personas a descubrir cuál es la suya y a tomar el control de esta. El desarrollo de la marca para una persona es un trabajo continuo, y hay pasos para crear una que sea verdadera y sólida. La esencia de esto es saber qué impulsa a la persona, incluidos sus valores y pasiones en la vida.

Cada persona ya es una marca y de ella depende qué hacer para construirla, cambiarla y mejorarla. En tiempos de incertidumbre, una marca, y su reputación, debe hablar por sí misma.

Para Henderson (2019), el personal branding es muy importante porque ayuda a establecer un proceso para desarrollar y mantener, de forma continua, la buena reputación e imagen de una persona ante sus diferentes stakeholders. 
Por otra parte, queda claro que a través del personal branding se construye la marca personal, que se define así:

Un proceso consciente y deliberado por parte de una persona, orientado a dejar una huella mostrando el valor diferencial que puede ofrecer a terceros, mediante la generación de confianza. Lleva el adjetivo «personal» porque parte de un esfuerzo de la persona por destacar sus atributos, pero en realidad el objetivo último es capitalizar esa huella de alguna forma en activos tangibles dentro de lo profesional, ya sea con nuevas ofertas laborales, con proyectos propios o con un aumento de la empleabilidad (Lara, 2019).

En este enfoque puede apreciarse la prioridad de la acción conducente a resultados; aunque estos también están presentes, partiendo de la propia huella (el primero de ellos) y sus diversos efectos.

Gestionar una marca personal es, esencialmente, trabajar de forma sistémica, estratégica y sistemática, con un enfoque en los procesos, para que esta logre, desarrolle y conserve un posicionamiento positivo en la mente de otras personas que integran, eventual, temporal o permanentemente, su entorno humano. Por lo general, dicho posicionamiento es un resultado asociado al marketing; véase a continuación su tratamiento en el personal marketing, desde diversas posiciones.

\section{El personal marketing: hacia el posicionamiento competitivo de la marca personal}

El marketing no solo es útil para productos o servicios: también puede y debe aplicarse a las personas y su actividad profesional y social, con un equilibrio adecuado entre tales dimensiones. Al conjunto de estrategias, métodos, técnicas y prácticas utilizadas con esos fines se le conoce como marketing personal, o personal marketing, en inglés.

Así, en el sentido laboral-profesional,

Un plan de marketing personal es para gente sanamente ambiciosa que quiere lograr un puesto de trabajo; desempeñarlo bien; reorientar su carrera cuando lo estime oportuno; y alcanzar, o al menos perseguir la excelencia, dando un servicio a la sociedad y siendo feliz con ello (Acosta, 2006, 17).

La lógica del personal marketing se relaciona con las formas de dar a conocer la marca de los individuos en los entornos laborales, de desarrollo y desempeño profesional en los que una persona está o pretende ubicarse. 
Como lo explica Carrillo $(2019,74)$ :

[...] el marketing personal, como transferencia del enfoque organizacional al individual, hace necesario que los individuos analicen su entorno, clientes, competencia, y realidad social, principalmente para organizar dichas actividades para alcanzar sus metas que tendrán que contemplar a los clientes.

Al relacionar el personal marketing con el personal branding, dado que su centro común es la marca personal, cabe analizarlo como el proceso estratégico de definir, desarrollar y comunicar para lograr un posicionamiento único de un individuo, con base en sus cualidades y fortalezas, con actividades e iniciativas diferenciadas y únicas para cada persona (Balula y Vasconcelos, 2020).

La construcción de una marca personal engloba el automarketing (self-marketing) y el autobranding (self-branding), poniendo así un fuerte foco en la promoción de las personas en el proceso de creación de una imagen profesional para postularse a un puesto de trabajo o progresar en un área profesional específica. (Potgieter y Doubell, 2018).

Si se aplica el caso del siempre vigente modelo de las $4 \mathrm{P}$, puede plantearse que el marketing personal gestiona el sistema producto-precio-plaza-promoción que está asociado con la persona, lo que es, realiza y aporta a la sociedad, y la marca personal es aquella en la que se convierte la persona en cuestión para sus diferentes públicos, a partir de todo lo anterior. Esto es, extrapolando al tema la lógica mercadotécnica empresarial aplicable a la marca personal (Peters,1999).

Shepherd (2010) considera que el marketing personal o automarketing consiste en diversas actividades emprendidas por individuos para hacerse visibles en el mercado, aumentando su exposición. Las estrategias de marketing personal son complejas, multidimensionales y dinámicas, y se anima a las personas a que realicen esas actividades por sí mismas.

Parece más resaltable la posibilidad de vender el conjunto de beneficios profesionales y humanos que alguien puede obtener al relacionarse, o trabajar otros, colocándolos ante sus ojos como una opción competitiva entre las disponibles, y no únicamente venderse como personas. Se coincide así con Pérez Ortega (2008), quien propone que la marca personal no es la venta de una persona, sino todo lo contrario; se enfoca en destacar las fortalezas laborales y personales $y$, a través de estas acciones, posicionar a la persona. 
De este modo, las acciones y los resultados en la organización o en el entorno social serán los que reflejen a la persona y propicien un posicionamiento favorable de su marca personal.

Como lo indica Soriano (2011), el marketing personal es la adaptación del marketing al individuo, que ofrece a las personas diferentes herramientas para promocionarlo y así lograr sus objetivos (por ejemplo, conseguir un trabajo).

Los beneficios de contar con un plan de marketing personal son:

- Darse la oportunidad de hacer un análisis de la situación de la persona.

- Detectar fortalezas y oportunidades para realizar un planteamiento estratégico que capitalice las fortalezas y disminuya las áreas de oportunidad detectadas.

- Plantear objetivos, estrategias y tácticas que sumen a la construcción del posicionamiento de la persona y a que logre sus objetivos de vida.

- Al momento de elegir actividades, priorizar aquellas que sumen a sus objetivos.

- Al tener métricas claras, será más sencillo ver los avances en el logro de los resultados esperados.

Estas ventajas son evidentes para Chaparro et al. (2019), quienes plantean que, así como las empresas comunican los beneficios de sus productos y servicios, de la misma manera debe hacerse con los profesionistas, seleccionando cuáles son sus competencias, cualidades y ventajas competitivas, para que con base en las mismas, comuniquen su posicionamiento.

El marketing personal es la dirección y administración de las actividades de mercadotecnia para la construcción de la marca personal; además, define estándares y procedimientos para lograr efectividad en su posicionamiento, como resultado de hacerla visible de la forma correcta para los públicos adecuados e impactarlos positivamente en los temas pertinentes.

En este orden de ideas, ElMassah et al. (2019) y Lair et al. (2005) mencionan que la marca personal va más allá de una estrategia simple y necesaria para que las personas puedan negociar un entorno económico turbulento. En el mismo sentido, afirman: «Atrás quedaron los días en los que el valor para su empresa o sus clientes proviene únicamente de sus ofertas. Hoy en día, la gente quiere comprar promesas de valor únicas de marca» (Arruda, 2002, 5). 
Por su parte, Ruiz et al. (2020) y Facchin (2018) nos sugieren el uso de redes sociales y plataformas en línea como herramientas para la construcción de la marca personal. Además de ser muy rentables, permiten medir fácilmente los resultados de las iniciativas implementadas.

\section{La importancia del personal marketing y personal branding en las organizaciones}

Según Recolons (2021b), la administración de la marca personal en las organizaciones tiene diferentes ventajas, entre las que menciona y describe la humanización de las marcas, generar confianza o recuperar la que se ha perdido, la alineación de los valores personales y corporativos en una sola voz, la amplificación del mensaje organizacional, el apoyo a la entidad en entornos de crisis, la retención del talento, la diferenciación, el networking y la posible generación de un plan B para los colaboradores, si fuese necesario.

Todo ello da lugar a importantes refuerzos para la marca corporativa, razón por la cual, según Amoako y Okpattah (2018), los reclutadores buscan candidatos que conozcan sus fortalezas y cómo aprovecharlas de manera adecuada en beneficio de la empresa. Por lo tanto, al destacar y presentar claramente su marca personal, cuentan su historia y la ajustan a las expectativas del reclutador.

Mientras más fuertes e influyentes sean las marcas personales de los colaboradores, mayor y mejor será la imagen organizacional, el impacto de la marca corporativa, así como las oportunidades que puedan emerger a nivel individual y organizativo.

En este orden de ideas, Yanzhen et al. (2021) proponen que el efecto persuasivo funciona creando deseo a través del desarrollo y la percepción de la marca. En un mercado laboral, los compradores son empleadores que buscan candidatos y los productos son candidatos para el puesto. Para los gerentes y directivos, el personal branding probablemente sea aún más importante, dado que la marca personal ya no es una opción, es un poderoso facilitador de liderazgo. De hecho, el personal branding en las redes sociales es un compromiso de tiempo completo con el viaje de definir a una persona como líder y debe considerarse cuidadosamente antes de emprender este camino. Por lo mismo, conviene un plan de mercadotecnia personal para los directivos clave de las empresas, pues son piezas fundamentales para la construcción de la reputación corporativa. 
Las empresas se han percatado de la importancia de trabajar también de forma estratégica la imagen de sus directores generales y colaboradores, como parte de la construcción de una buena imagen corporativa. Ello suele fluir a través de la creación de planes de marketing personal para sus directivos, alineados a las actividades de branding de las organizaciones que dirigen. Todo lo planteado genera, como una de sus consecuencias favorables, el posicionamiento positivo de las marcas personales de los involucrados, en el mercado, entre los clientes de la entidad y ante los demás stakeholders que interesan a la mismas.

De acuerdo con Tekin (2007), el director general o CEO (Chief Executive Officer) es el responsable del éxito o fracaso de una corporación, al influir favorablemente no solo en el logro de las ganancias de la empresa, sino también en el desempeño de los empleados y en la creación de valor que esta dirige a los accionistas y a la sociedad. De esta manera, el personal branding de los directores debe de estar ligado a los valores y características de las empresas que lideran (Wang et al., 2011).

Un director es el guardián designado de una empresa y está relacionado con los resultados de su reputación corporativa (Turk et al., 2012). De acuerdo con Buitrago y León (2018), se considera al líder empresarial idóneo a partir de su influencia como marca personal, con características tales como interés por el tema y postura clara y firme al respecto, capacidad de interacción y de convocatoria, reconocimiento social, intereses comunes con el grupo, y capacidad de compartir y debatir sus ideas.

A modo de ejemplo, esto puede comprobarse en el último estudio de Monitor Empresarial de Reputación Corporativa (Merco México, 2020), que coincide en que, en México, las principales empresas con mejor reputación corporativa de alimentos, bebidas y bancos cuentan con líderes también evaluados con una reputación personal muy positiva, como Daniel Servitje, de Grupo Bimbo, José Antonio Fernández Carbajal, de Femsa, Eduardo Osuna, de BBVA, y Ernesto Torres Cantú, de Citibanamex. En 2019, Grupo Bimbo también había ocupado la primera posición de empresa y líder con mejor reputación corporativa.

Lo anterior no es exclusivo del caso mexicano. Esto se confirma, por poner un ejemplo, en el estudio de Flores et al. (2018), quienes en su investigación en República Dominicana asocian la marca personal de un alcalde y la marca corporativa del ayuntamiento dirigido por él.

Lo planteado hasta aquí sustenta la idea de que, para lograr una buena imagen empresarial, además de crear planes sólidos de marketing corporativo, se debe trabajar en planes igualmente sólidos de personal branding y personal marketing para los equipos directivos. 


\section{Metodología}

\section{a) Estudio exploratorio:}

Con base en un estudio exploratorio de encuestas digitales a través de un formulario que se diseñó en Google, se efectuó un análisis de la información, utilizando el modelo de clasificación de red neuronal, cuyo objetivo era conocer la percepción y el conocimiento que los participantes tenían del concepto «marca personal». La encuesta contenía preguntas de opción múltiple, dicotómicas y abiertas, con el fin de que los encuestados pudieran describir lo que pensaban al respecto.

Se utilizó una técnica de muestreo no probabilístico, ya que la encuesta fue aplicada a personas interesadas en participar en el primer evento de marca personal realizado en México, organizado por Integra, la primera agencia de personal branding en el país, o que tuvieran interés sobre el tema.

Fases del análisis de la información:

- Para poder realizar la base de conocimiento se usaron los datos de una encuesta, mediante la herramienta de Google, a 112 personas, 45 \% hombres y $55 \%$ mujeres, de 21 a 50 años, con diferentes ocupaciones que iban desde estudiantes hasta empleados.

- Las encuestas fueron aplicadas durante 2018 por Integra, que aportó la base de datos de las respuestas para analizarlas mediante el programa de SPSS.

- Para analizar el conocimiento que se tiene de la marca personal, los objetivos de las preguntas seleccionadas se enfocaron en:

- Identificar el nivel de conocimiento del concepto de marca personal.

- Determinar la importancia que se concede a la marca personal.

- Definir cuáles son los atributos que valoran más de la marca personal.

- La pregunta: ¿qué importancia tiene para usted su marca personal?, indica qué tan importante es la marca personal para el encuestado; en un principio este dato era numérico, pero se cambió en alta, media y baja, con base en los resultados. Es la variable predictiva.

b) Investigación bibliográfica

Fue elaborada mediante la revisión de literatura enfocada esencialmente en el contraste y el análisis conceptual, en la valoración de propuestas conceptuales y 
analíticas, en el análisis de los enfoques propuestos y en la crítica científica cuando fue requerida. El objetivo: profundizar en la relevancia de la marca personal e impulsar su posicionamiento y construcción a través de las herramientas de personal branding y personal marketing como procesos complementarios para generar marcas personales sólidas y consistentes, que sumen a las carreras profesionales de los seres humanos y a la reputación corporativa de las empresas, a saber manejar adecuadamente las marcas personales de sus directivos y colaboradores.

Las fuentes se clasificaron con un enfoque general, de la forma siguiente:

- Textos de índole académica: artículos, tesis doctorales y de maestría, libros especializados en el tema como resultado de investigación o de experiencia autoral, publicados durante los tres años más recientes (2018, 2019, 2020 y lo que ha transcurrido hasta junio de 2021).

- Textos de índole académica: artículos, tesis doctorales y de maestría, libros especializados en el tema como resultado de investigación o de experiencia autoral, publicados en épocas anteriores a los tres años más recientes, pero con importancia y trascendencia en el tema.

- Artículos pertinentes que procedan de blogs especializados en el tema, con al menos tres años de trayectoria publicando sistemáticamente sobre este y cuyos autores gozan de prestigio profesional en el campo.

- Informes especializados sobre temas relacionados con los conceptos tratados en el artículo.

Los conceptos analizados fueron los siguientes:

- Marca personal, personal branding y personal marketing

Las relaciones y conexiones interconceptuales analizadas fueron las siguientes:

- Relación entre marca personal y personal branding, relación entre personal branding y personal marketing, y significados de las conexiones interconceptuales.

Para desarrollar la metodología, se siguieron los siguientes pasos:

1. Identificación de los conceptos clave y las principales tendencias que actualmente marcan el desarrollo del debate internacional acerca del tema.

2. Análisis del tratamiento de los conceptos y tendencias en una muestra de 45 fuentes ya recopiladas y en estudio, equivalentes al $100 \%$ de las fuentes revisadas (universo), divididas del modo ya mencionado. En la tabla 1 se realiza una 
revisión de literatura de los conceptos de marca personal, personal branding y personal marketing y se desglosan las fuentes por tipo, cantidad y fecha de publicación (ver tabla 1).

Tabla 1. Revisión de literatura de los conceptos de marca personal, personal branding y personal marketing

\begin{tabular}{|c|c|c|c|}
\hline$\#$ & Tipo de fuente & Cantidad & Período de publicación \\
\hline 1 & Tesis doctorales sobre el tema & 4 & $\begin{array}{l}1 \text { en } 2014 \\
1 \text { en } 2015 \\
2 \text { en } 2019\end{array}$ \\
\hline 2 & Tesis de maestría sobre el tema & 1 & 1 en 2018 \\
\hline 3 & $\begin{array}{l}\text { Libros impresos y digitales especializa- } \\
\text { dos en el tema }\end{array}$ & 15 & $\begin{array}{l}1 \text { en } 1999 \\
1 \text { en } 2000 \\
1 \text { en } 2001 \\
1 \text { en } 2006 \\
1 \text { en } 2009 \\
1 \text { en } 2011 \\
1 \text { en } 2014 \\
2 \text { en } 2016 \\
1 \text { en } 2018 \\
4 \text { en } 2020 \\
1 \text { en } 2021\end{array}$ \\
\hline 4 & $\begin{array}{l}\text { Artículos que tratan el tema, publica- } \\
\text { dos en medios indexados en bases de } \\
\text { datos académicas }\end{array}$ & 16 & $\begin{array}{c}1 \text { en } 2005 \\
1 \text { en } 2007 \\
1 \text { en } 2011 \\
1 \text { en } 2012 \\
12 \text { entre } 2018 \text { y } 2021\end{array}$ \\
\hline 5 & $\begin{array}{l}\text { Ensayos y artículos de análisis, de opi- } \\
\text { nión y de divulgación acerca del tema, } \\
\text { publicados en medios no indexados } \\
\text { en bases de datos académicas }\end{array}$ & 8 & $\begin{array}{l}1 \text { en } 2011 \\
1 \text { en } 2012 \\
3 \text { en } 2017 \\
1 \text { en } 2019 \\
1 \text { en } 2021\end{array}$ \\
\hline 6 & $\begin{array}{l}\text { Informe nacional sobre el ranking } \\
\text { empresarial y de liderazgo, pertinente } \\
\text { al tema }\end{array}$ & 1 & 1 en 2020 \\
\hline
\end{tabular}

Fuente: elaboración propia con base en la bibliografía de este documento. 


\section{Resultados}

a) Análisis del estudio exploratorio

i. Conocimiento de marca personal

Las personas tienen una idea de lo que es la marca personal, sin poder describirla adecuadamente. Se le asocia con un tema de percepción, venderse, estrategias, diferenciación e imagen, principalmente (ver gráfica 1).

Gráfica 1. ¿Qué es marca personal para ti?

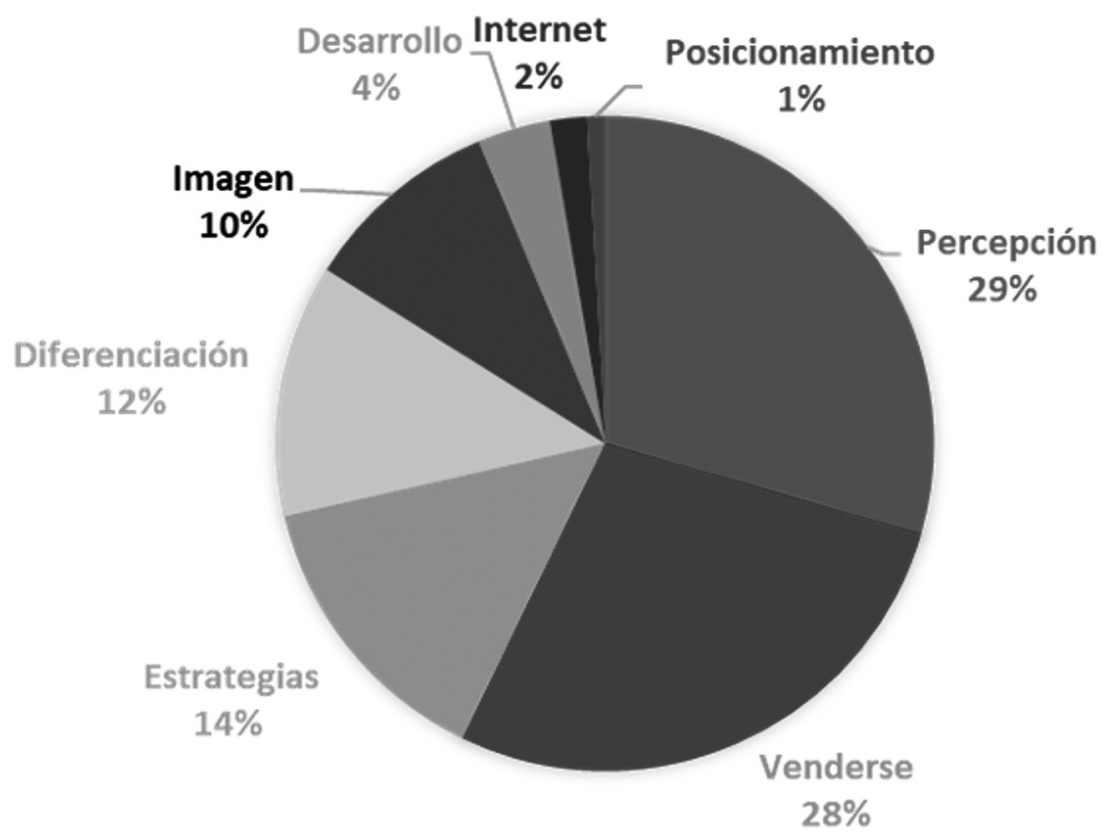

Fuente: elaboración propia con base en el estudio exploratorio.

ii. Importancia de la marca personal

Es un concepto relevante, aunque las personas no sepan con certeza la manera en que pueden construir su marca personal, lo que refleja un gran potencial para difundir los beneficios de trabajar estratégicamente en su construcción (ver gráfica 2). 
Gráfica 2. ¿Qué tan importarte es la marca personal para ti?

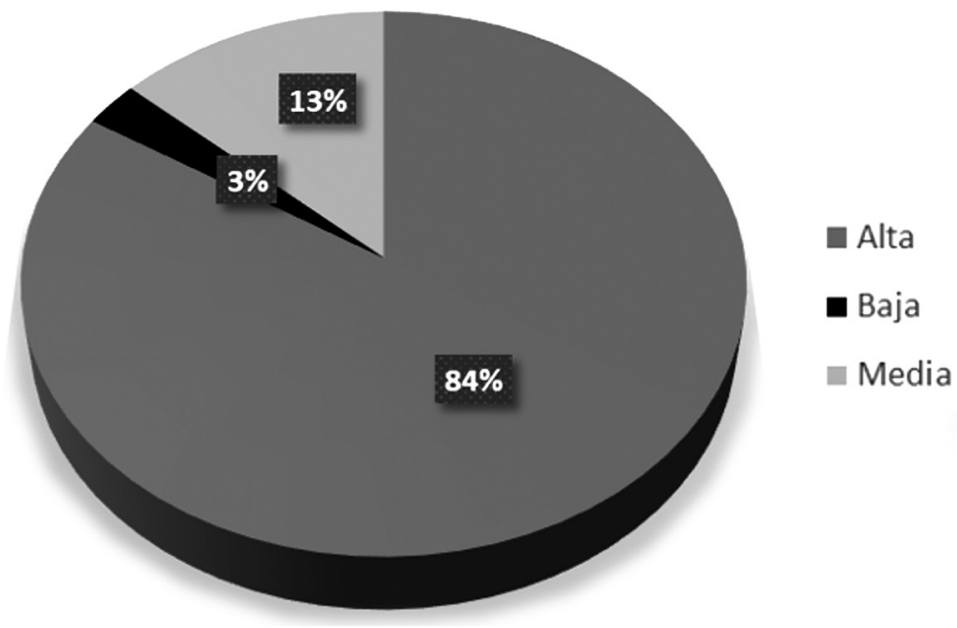

Fuente: elaboración propia con base en el estudio exploratorio.

iii. Significado del concepto de marca personal

Uno de los temas que no sale en el momento de describir la marca personal es el autoconocimiento, uno de los puntos más importantes al generar estrategias de personal branding (ver tabla 2).

Tabla 2. ¿Qué es la marca personal para ti?

\begin{tabular}{|l|r|}
\hline Alta & $\mathbf{8 4} \%$ \\
\hline Percepción & $28 \%$ \\
\hline Venderse & $24 \%$ \\
\hline Estrategias & $17 \%$ \\
\hline Diferenciación & $13 \%$ \\
\hline Imagen & $12 \%$ \\
\hline Desarrollo & $3 \%$ \\
\hline Internet & $2 \%$ \\
\hline Posicionamiento & $1 \%$ \\
\hline
\end{tabular}


(continúa)

\begin{tabular}{|l|r|}
\hline Media & $\mathbf{1 3 \%}$ \\
\hline Percepción & $47 \%$ \\
\hline Venderse & $33 \%$ \\
\hline Diferenciación & $13 \%$ \\
\hline Desarrollo & $7 \%$ \\
\hline Baja & $\mathbf{3} \%$ \\
\hline Venderse & $100 \%$ \\
\hline
\end{tabular}

Fuente: elaboración propia con base en el estudio exploratorio.

iv. Puntos de contacto con el concepto de marca personal

Finalmente, es importante mencionar que las personas han escuchado o se han enterado del concepto de marca personal principalmente por internet. Ello refuerza que las estrategias de difusión del concepto y de planes estratégicos para la construcción de la marca personal en herramientas digitales podría ser el medio más efectivo para la promoción y captación de individuos y empresas interesados en desarrollar planes de personal branding y de personal marketing (ver gráfica 3).

Gráfica 3. ¿Dónde has visto o escuchado acerca de marca personal?

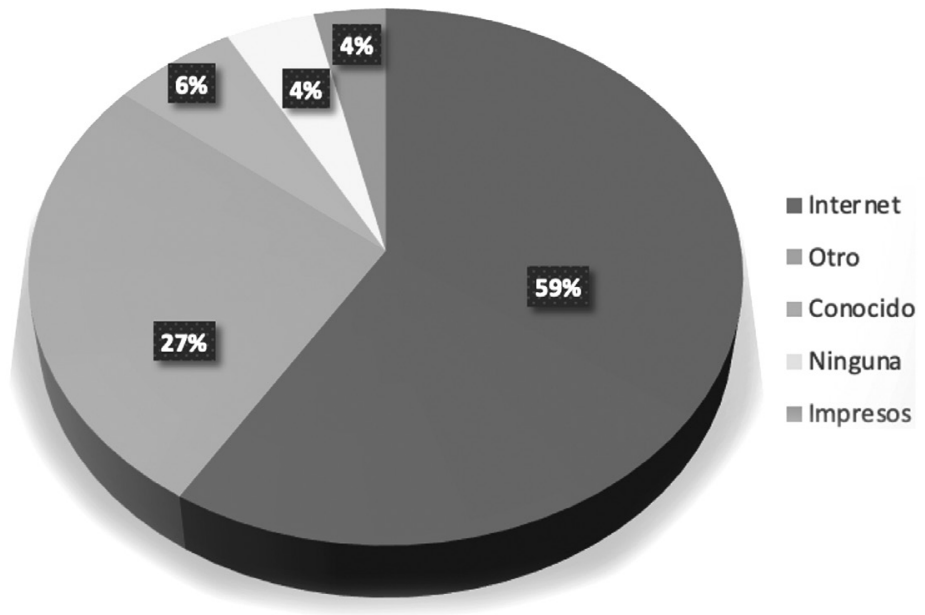

Fuente: elaboración propia con base en el estudio exploratorio. 
b) Investigación bibliográfica

Tras analizar el conjunto de las 45 fuentes consultadas y citadas en este artículo, en relación con sus objetivos, se pueden exponer los siguientes resultados:

i. Por lo general, la marca personal se aborda como un resultado de la gestión efectuada por el individuo con base en su persona y sus aportaciones a la sociedad.

ii. Se identificaron tendencias como las siguientes:

- A la digitalización de la gestión de marca personal, para efectuar con efectividad los procesos de personal branding y personal marketing.

- Al crecimiento paulatino de la investigación y la práctica para desarrollar y administrar la marca personal.

- A la integración y sistematización de estos conceptos (libros que abordan los temas de forma completa y generalizadora).

iii. Se aprecia un amplio consenso autoral en temas como los que se mencionan a continuación:

- El creciente significado de la marca personal en la esfera laboral y profesional, como factor clave para elecciones y decisiones.

- La necesidad de posicionar la marca personal a través del enfoque integrado del personal branding y el personal marketing.

- La importancia de administrar la marca personal, para evitar sesgos o malas interpretaciones de lo que se pretende proyectar al entorno.

- La necesidad de un método y técnicas de gestión para evitar improvisaciones que pueden ser contraproducentes.

- La importancia creciente del entorno digital para el personal branding y el personal marketing.

- La importancia de la integración entre el branding y el marketing en el nivel personal, con el branding y el marketing a nivel corporativo.

iv. Se propone y desarrolla un modelo para posicionar la marca personal mediante el personal branding y personal marketing (ver la figura 1). 
Fabiola Patricia Melchor Rueda, Enriqueta del Carmen Márquez Sánchez, Vladimir Deléyade Estrada Portales

Personal branding y personal marketing: procesos complementarios centrados en la marca personal Personal branding and personal marketing: complementary processes focused on the personal brand

Figura 1. Modelo para posicionar la marca personal mediante personal branding y personal marketing

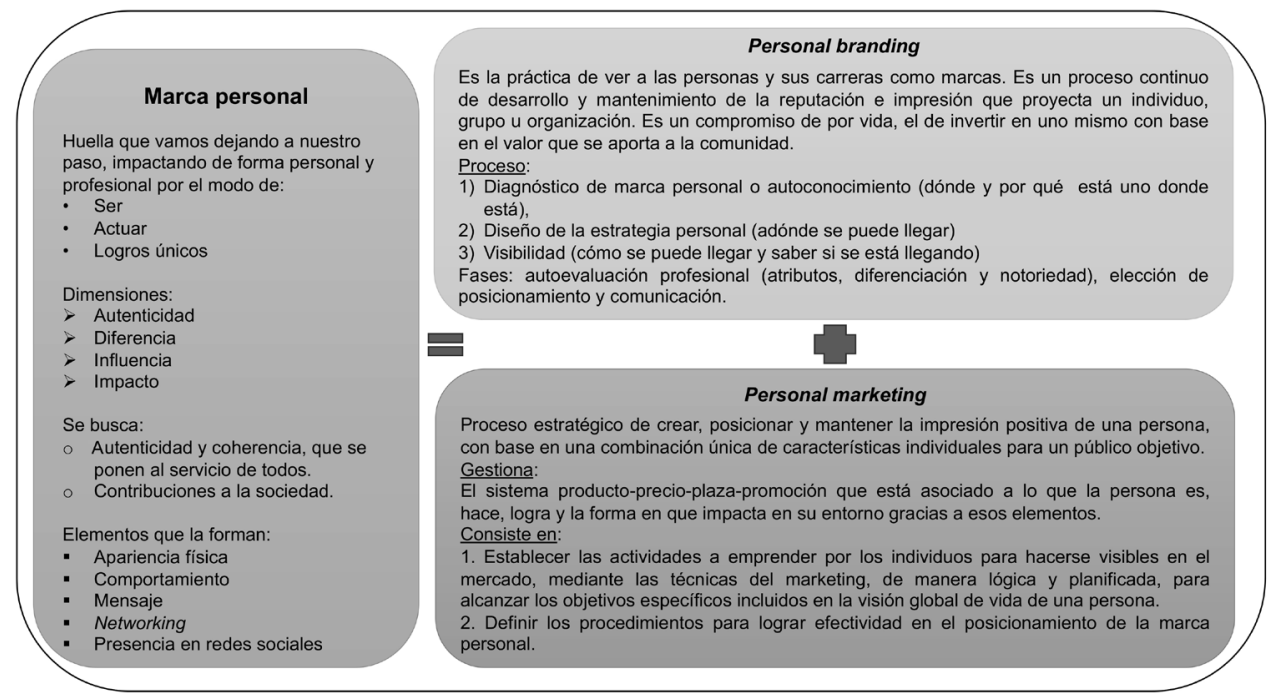

Fuente: elaboración propia con base en Buitrago y León (2018), Molares-Cardoso et al. (2020), Henderson (2019), Recolons (2021b), Peters (1997 y 1999), Pérez Ortega (2008), Balula y Vasconcelos (2020), Shepherd (2010) y Soriano (2011).

\section{Conclusiones}

La marca personal es al mismo tiempo el resultado del proceso de branding personal y el objetivo del proceso de marketing personal: al hacer personal branding se desarrolla, mejora y posiciona la marca, y al hacer marketing personal, esta se ubica en el entorno competitivo como una de las opciones disponibles. Mientras mejor sea la calidad del branding efectuado, será mucho más viable y efectivo el marketing a efectuar, pues el «producto» tendrá mayor valor intrínseco y percibido a los ojos de sus públicos. Estas tres categorías de gestión ameritan mayor estudio e investigación académica y práctica, para contribuir a los procesos de desarrollo personal y social en este nuevo y cada vez más exigente cambio de época.

Con base en el estudio exploratorio, se comprueba lo relevante del concepto de marca personal y el potencial que existe para difundir los beneficios de trabajar estratégicamente en la construcción de la misma. Con base en la investigación 
bibliográfica, se desarrolla un modelo para posicionar la marca personal, usando personal branding y personal marketing. En este modelo se indica el proceso, fases y formas de gestionar la marca personal con las dimensiones y elementos que la integran.

A lo largo de este documento, se corrobora que la construcción de la marca personal, es un proceso estratégico, estructurado, que requiere un trabajo continuo, coherente, con aportaciones de valor al individuo y a la sociedad. Cada día es más relevante para el crecimiento profesional de las personas y para mantener una reputación corporativa con una imagen positiva en las empresas.

Como futuras líneas de investigación, se considera importante analizar en detalle la conexión sistémico-estratégica entre el personal branding y el personal marketing, las metodologías de intervención profesional en ambos procesos, los métodos y las técnicas de investigación más pertinentes para su desarrollo, la producción intelectual en diferentes ámbitos, como contribución al conocimiento masivo de estos conceptos y procesos, así como el rol del entorno organizacional como espacio fundamental para la administración y posicionamiento de la marca personal.

Otra futura línea de investigación es el análisis de cómo permean las iniciativas de personal branding y personal marketing en las diferentes generaciones, y en cuál de estas se tiene una mejor aceptación, considerando que actualmente cuatro generaciones forman parte de la fuerza laboral. Los baby boomers aún se encuentran trabajando; los miembros de la generación $\mathrm{X}$ ocupan cargos de liderazgo; las personas de la generación $Y$ representan la mayor parte de la fuerza laboral, mientras que los miembros de la generación $Z$ forman parte de las personas que aún están incursionando en el mundo del trabajo (Kotler et al., 2021). 


\section{Referencias bibliográficas}

Acosta, J. (2006). Marketing Personal. El camino al éxito. ESIC.

Amoako, G. K. y Okpattah, B. K. (2018). «Unleashing salesforce performance: The impacts of personal branding and technology in an emerging market». Technology in Society, vol. 54, 2018. https://doi.org/10.1016/j.techsoc.2018.01.013

Arruda, W. (2002). «An Introduction to Personal Branding: A Revolution in the way we manage our careers». Reach Communications Consulting, Inc.

Balula, A. y Vasconcelos, S. (2020). «Personal Branding and storytelling: tales from the Tourism ESP classroom». Journal of Tourism \& Development, núm. 34, 2020.

Buitrago Acuña, R. y León Monque, M. (2018). «Branding personal como eje dinamizador de la imagen política para los candidatos a cargos de elección popular en Venezuela». Cuadernos Latinoamericanos de administración, vol.14, núm. 27, 2018.

Carrillo González, F. (2019). «Influencia del proyecto profesional y de la marca personal en la empleabilidad. Diseño, desarrollo y evaluación de la efectividad de un programa». Tesis de doctorado, Universidad de Zaragoza. Repositorio de la Universidad de Zaragoza-Zaguan.

Carrillo González, F. (2021). «Efectividad de un programa para la mejora del proyecto profesional y la marca personal». Revista Española de Orientación y Psicopedagogía, vol. 32 núm.2, 2021.

Chaparro Guevara, R., Ospina Estupiñan, H. y Alonso González, A. (2019). «Personal branding como elemento diferenciador en la figura de los docentes de instituciones de educación superior en Latinoamérica». Cuadernos Latinoamericanos de Administración, vol. 15, núm. 29, 2019.

Collell, J. (2019). «Guía práctica para gestionar tu marca personal». Jordi Collell [blog]. https://www.jordicollell.com/guia-practica-para-gestionar-tu-marca-personal/

Del Toro Acosta, A. (2015). «Marca personal en medios sociales digitales: propuesta de un modelo de autogestión». Tesis de doctorado, Universidad Complutense de Madrid. Repositorio Institucional de la UCM.

ElMassah, S., Michael, I., James, R. y Ghimpu, I. (2019) «An assessment of the influence of personal branding on financing entrepreneurial ventures». Heliyon, vol. 5, núm. 2, 2019. https://doi.org/10.1016/j.heliyon.2019.e01164

Estrada Portales, V.D. (2020a). Marca personal y empresa: la otra verdad. Editora Digital Lulu.

Estrada Portales, V.D. (2020b). ¿Se puede «vivir de la marca personal»? Editora Digital Lulu.

Facchin, J. (2018). «Imagen y Reputación Corporativa. Este Escaparate Ilamado Internet». El Blog de José Facchin [blog]. https://josefacchin.com/este-escaparatellamado-internet-imagen-y-reputacion-corporativa/ 
Fernández Gómez, J., Hernández-Santaolalla, V. y Sanz-Marcos, P. (2018). «Influencers, marca personal e ideología política en Twitter». Cuadernos.info. Comunicación y medios en Iberoamérica, núm. 42. https://doi.org/10.7764/cdi.42.1348

Flores, J. G., Barranco, M. y Díaz, Jenny. (2018). Comunicación y marketing municipal en entornos digitales. Caso: alcaldía de Santiago de los caballeros, período agosto 2016-abril 2018. Tesis de maestría, Universidad Abierta para Adultos.

Henderson, G. (7 de noviembre de 2019), «What Is Personal Branding?». Digital marketing blog [blog]. Digital Marketing.org

Kotler, P., Kartajaya, H. y Setiawan, I. (2021). Marketing 5.0: Technology for Humanity. Wiley.

Lair, D., Sullivan, K. y Cheney, G. (2005). «Marketing and the recasting of the professional self: the rhetoric and ethics of personal branding». Management Communication Quarterly, vol. 18, núm. 3, 2005. https://doi.org/10.1177/0893318904270744

Lara Padilla, T. (2019). «La construcción de marca personal del periodista: del blog a Twitter (2004-2019)». Tesis de doctorado, Universidad Complutense de Madrid. Repositorio Institucional de la UCM.

Monitor Empresarial de Reputación Corporativa, Merco México (26 de agosto de 2020), «Las 100 empresas y los 100 líderes con mejor reputación en México» (comunicado de prensa), Merco. https://www.merco.info/files/2020/08/983/nota-de-prensamerco-empresas-y-lideres-mexico-2020.pdf

Molares-Cardoso, J., López de Aguileta, C. y Legerén Lago, B. (2020). «A importância do personal branding como elemento de relações públicas: uma experiência de avaliação internacional». Comunicação e Sociedade, 19-35. https://doi.org/10.17231/ comsoc.0(2020).2738

Park, J., Williams, A. y Son, S. (2020). «Social Media as a Personal Branding Tool: A Qualitative Study of Student-Athletes' Perceptions and Behaviors». Journal of Athlete Development and Experience, JADE, vol. 2, núm.1, 2020.

Pérez Ortega, A. (2008). Marca Personal: cómo convertirse en la opción preferente. ESIC. Pérez Ortega, A. (2014). Marca personal para dummies. Planeta de libros.

Pérez, C. y Gringarten, H. (2020). «Personal Branding: An Essential Choice in the COVID-19 Era?». Journal of Multidisciplinary Research, vol. 12, núm. 2, 2020.

Peters, T. (1997). «The brand called You». Fast Company, vol. 10, núm. 10.

Peters, T. (1999). 50 claves para hacer de usted una marca. Ediciones Deusto.

Potgieter, A. y Doubell, M. (2018). «Authentic personal branding is not your social media page». International Journal of Contemporary Management, vol. 9, núm. 4, 2018.

Rampersad, H. (2009). Tu marca personal. LID Editorial Empresarial.

Real Academia Española. (2014). «Marcar». Diccionario de la lengua española (23a ed.).

https://dle.rae.es/marcar 
Recolons, G. (2020). Si no aportas, no importas. Rasche.

Recolons, G. (26 de junio de 2021a). «Una marca llamada tú, remix 2021». Guillem Recolons [blog]. https://www.guillemrecolons.com/una-marca-llamada-tu-remix-2021/

Recolons, G. (11 de septiembre de 2021b). «Personal branding en la empresa: aplicaciones, pros y contras». Guillem Recolons [blog]. https://www.guillemrecolons. com/personal-branding-empresa/

Regalado-Ortegón, A. (2018). «Branding personal y creación de gurús académicos». Cuadernos Latinoamericanos de Administración, vol. 14, núm. 26, 2018.

Ries, A. y Ries, L. (2001). Las 22 leyes inmutables de la marca cómo convertir un producto o un servicio en una marca mundial. McGraw-Hill.

Ruiz Contreras, A. B., Rodríguez Suárez, C., Juárez Pérez, S. y Mascarúa Alcázar, M. A. (2020). «Marketing de contenido online, compromiso con el cliente, imagen pública y reputación corporativa de las MYPES de Tehuacán». CPMark. Cuaderno Profesional de Marketing, vol. 8, núm.4, 2020.

Sagredo, Elena (2 de marzo de 2012). «Un CEO no puede no tener una marca personal». Canal CEO.

Shepherd, I. (2010). «From cattle and coke to Charlie: Meeting the challenge of selfmarketing and personal branding». Journal of Marketing Management, vol. 21, 2005. https://doi.org/10.1362/0267257054307381

Soriano, C. (2011). Plan de Marketing Personal. Ediciones Díaz de Santos.

Tekin, Y. (2007). A modern leadership approach visionary leadership: A research in accommodation facilities with 5 stars carrying on business in Antalya. Akdeniz University, Institute of Social Sciences.

Turk, J.V., Jin, Y., Stewart, S., Kim, J. y Hipple, J.R. (2012). «Examining the interplay of an organization's prior reputation, CEO's visibility, and immediate response to a crisis». Public Relations Review, vol. 38, núm. 4, 2012. https://doi.org/10.1016/j. pubrev.2012.06.012

Wang, H., Tsui, A.S. y Xin, K.R. (2011). «CEO leadership behaviors, organizational performance, and employees attitudes». The Leadership Quarterly, vol. 22, núm. 1, 2011. https://doi.org/10.1016/j.leaqua.2010.12.009

Yanzhen, C., Huaxia, R. y Whinston, A. B. (2021). «Tweet to the Top? Social Media Personal Branding and Career Outcomes». MIS Quarterly, vol. 45, núm. 2, 2021. https://doi.org/10.25300/MISQ/2021/14617 


\section{Sobre los autores}

Fabiola Patricia Melchor Rueda es doctoranda en Mercadotecnia y Comunicación Estratégica por la Universidad Anáhuac del Norte y estudió la maestría en Economía y Negocios en la misma universidad. Es licenciada en Mercadotecnia con mención honorífica de la Universidad Tecnológica de México. Tiene más de 18 años de experiencia en marketing en empresas internacionales, trabajando en áreas como category management, trade marketing, marketing digital, alianzas estratégicas, campañas, entre otros. Actualmente es directora de Marketing y Comunicación de Lean Business Group.

fabiolamelchor@gmail.com

https://orcid.org/0000-0002-4267-2847

Enriqueta Márquez es doctora en Ciencias Empresariales especializada en Dirección Internacional de Empresas por la Universidad Antonio Nebrija en España. Tiene una maestría en Mercadotecnia y Publicidad de la Universidad Anáhuac y es egresada con Mención Honorífica de la licenciatura en Mercadotecnia del Instituto Tecnológico y de Estudios Superiores de Monterrey. Su experiencia inició en SONY Electrónicos y posteriormente se especializó en empresas de consumo como Conagra Foods, Herdez y Nestlé. Desde 2008 paralelamente dio inicio a su carrera docente como catedrática de la Universidad Anáhuac, en la maestría de Mercadotecnia Integral, de Planeación de Medios, el doctorado en Comunicación y Mercadotecnia Estratégica, y el doctorado en Liderazgo y Dirección de Instituciones de Educación Superior. Actualmente es también miembro del Consejo de la Facultad de Comunicación de la Universidad Anáhuac, así como fundadora y directora general de Trascendencia, empresa enfocada en brindar cursos y asesorías de Mercadotecnia a empresas nacionales e internacionales. Cuenta con más de 22 años de experiencia en el área de Mercadotecnia y 13 años impartiendo clases a nivel de maestría y doctorado.

enriquetamarquezs@gmail.com,enriqueta.marquezs@anahuac.mx https://orcid.org/0000-0002-9653-8950 
Vladimir Deléyade Estrada Portales es doctor en Desarrollo Gerencial y Organizacional, con maestría en Consultoría Gerencial; es licenciado en Educación y tiene posgrados en Administración y Dirección de Empresas, en Capacitación Gerencial y en Gestión de Recursos Humanos. Tiene 37 años de carrera profesional y académica, de los cuales 27 han transcurrido también en educación superior. Ha publicado nueve libros en formato digital y es coautor de otros seis, así como de más de 400 artículos, algunos de ellos publicados en prestigiosos medios internacionales. $\mathrm{Ha}$ asesorado alrededor de 400 tesis de grado y posgrado, y ha sido expositor en más de 30 congresos. Actualmente es profesor universitario en grado y posgrado, asesor académico, mentor profesional internacional, consultor gerencial, académico y de marca personal, conferenciante internacional, entrenador de productores intelectuales en formato escrito, blogger académico, embajador de la Red Global de Mentores en la República Dominicana, y primer embajador de Marca de la Red Social Profesional FEGO, dedicada a crear, compartir, encontrar y difundir publicaciones digitales personales y corporativas, con novedoso formato tecnológico. Investiga, forma, asesora y consulta sobre personal branding, gerencia y liderazgo, educación y academia, comunicación digital, blogging y mentoring.

mentor.asesor.academico@gmail.com

https://orcid.org/0000-0002-6816-4162 